\title{
Lessons from the National Cooperative Growth Study
}

David Wyatt

Department of Pediatrics, Medical College of Wisconsin, 8701 Watertown Plank Road, Milwaukee, WI 53226, USA

(Correspondence should be addressed to D Wyatt; Email: dwyatt@mail.mcw.edu)

\begin{abstract}
Objective: To review the National Cooperative Growth Study (NCGS), a national post-marketing surveillance program for children treated with biosynthetic growth hormone (GH) products from Genentech.

Methods: Representative data are presented to summarize the extensive experience of the NCGS. This study is a multicenter, observational surveillance registry begun in 1985 in coordination with the release of the first recombinant DNA biosynthetic GH.

Results: After almost 20 years, data from over 47000 patients representing 165000 patient years have been collected. There are over 12000 active subjects (estimated to be $\sim 75 \%$ of all current patients treated with a Genentech GH product) at 435 centers, providing extensive efficacy and safety data. The GrowTrak program is a secure, user-friendly database which encourages a high level of participation by the pediatric endocrine community in the USA. Efficacy has been shown in a variety of clinical diagnoses, including isolated (IGHD) and organic growth hormone deficiency (OGHD), idiopathic short stature (ISS), and Turner syndrome. Safety monitoring of this large population has provided reassuring evidence that leukemia (de novo or relapse), extracranial nonleukemic neoplasms and central nervous system (CNS) tumor recurrence are not associated with GH therapy. A small risk of intracranial hypertension and slipped capital femoral epiphyses has been suggested, especially in children with renal disease or Turner syndrome. NCGS substudies have also provided important insights into actual clinical practice. For example, screening for renal, cardiac, or auditory comorbidities in Turner syndrome is often done less than is recommended by national guidelines (NCGS 9). Furthermore, almost 65\% of children referred for short stature may be lost to follow-up before an evaluation is completed (NCGS 8).

Conclusions: The NCGS has proven to be a valuable method of monitoring the safety and efficacy of biosynthetic GH. The study has attracted wide physician participation due to the data collection software used and the extensive sharing of the analyses of that data with the providers.
\end{abstract}

European Journal of Endocrinology 151 S55-S59

\section{Introduction}

The National Cooperative Growth Study (NCGS) was begun in 1985 when Protropin, the recombinant DNA-based biosynthetic growth hormone (GH), became available for prescription use in children with GH deficiency (GHD). Since this was the first recombinant $\mathrm{GH}$ to be used, and since pituitary-derived $\mathrm{GH}$ had just been withdrawn in the USA because of the association with Creutzfeldt-Jakob encephalopathy, the Food and Drug Administration (FDA) and other regulatory agencies were particularly cautious about the introduction of this therapy. The NCGS was thus initially designed to respond to the safety-reporting requirements of these agencies. Though safety monitoring remains essential, the NCGS has evolved substantially beyond this initial goal (Table 1). It has become a valuable tool for the physicians who participate in the study, particularly since the great majority of children receiving GH products from Genentech (South
San Francisco, CA, USA) are enrolled. For almost 20 years, the NCGS has provided reassuring evidence of the safety and efficacy of GH. This review will present representative data from the NCGS.

\section{Methods and subjects}

The NCGS is a large-scale, multicenter, open-label, observational, post-marketing surveillance study of children treated with any of the GH products from Genentech (Nutropin AQ, Nutropin Depot, Nutropin and Protropin). It involves non-interventional monitoring of patients from the initiation to the conclusion of GH therapy. Where possible, post-treatment heights are obtained until epiphyseal closure occurs. Any physician treating children for growth failure with a Genentech product is invited to participate. Participating physicians obtain local institutional approval for enrolling patients anonymously in the NCGS. 
Table 1 Goals of the National Cooperative Growth Study.

Determine efficacy in a variety of patient populations Develop outcome predictors

Define the demographics of patients receiving therapy

Develop new inquiries about patient subpopulations (substudies)

Communicate findings with providers

Monitor safety of therapy in a large population

Data are collected with a database program (GrowTrak, South San Francisco, CA, USA), which resides on a computer at each provider's site. Complete patient data are maintained locally; de-identified data are encrypted and transmitted for central analysis. The on-site software provides local error correction prior to data submission, thus eliminating the need for post-submission corrections.

\section{Results}

Over $47000 \mathrm{GH}$-treated patients have been monitored since 1985, representing 165000 patient years. There are about 12000 children currently under therapy in the NCGS, representing approximately $75 \%$ of all children treated with Genentech GH. There are over 900 participating investigators at 435 sites in the USA and Canada. This extensive degree of monitoring provides a solid foundation for the analysis of the safety and efficacy of GH therapy in this patient population.

\section{Efficacy}

Extensive long-term data have confirmed the efficacy of GH therapy in a variety of clinical situations, including isolated growth hormone deficiency (IGHD) and organic growth hormone deficiency (OGHD), idiopathic short stature (ISS), Turner syndrome and chronic renal insufficiency (1). Mean growth rates at a standard dose of $0.3 \mathrm{mg} / \mathrm{kg}$ per week are similar in patients with IGHD and OGHD. While children with ISS had a less vigorous response in the first 2 years of therapy, they had a growth velocity equal to that of children with OGHD

Table 2 NCGS demographics - all patients. Change in percentage of treated population (new starts) by diagnosis.

\begin{tabular}{lcc}
\hline Diagnosis & $\mathbf{1 9 8 6}$ & $\mathbf{2 0 0 2}$ \\
\hline IGHD $^{1}$ & $39 \%(452)$ & $45 \%^{3}(1130)$ \\
OGHD & $26 \%(294)$ & $12 \%{ }^{3}(293)$ \\
ISS & $14 \%(164)$ & $19 \%{ }^{3}(475)$ \\
Turner syndrome & $7 \%(79)$ & $8 \%(190)$ \\
Renal, other & $14 \%(157)$ & $16 \%(388)$ \\
& $100 \%$ & $100 \%$ \\
\hline
\end{tabular}

$n$ for each diagnosis and year in parenthesis.

${ }^{1}$ Isolated growth hormone deficiency with no anatomic defect.

${ }^{2}$ Growth hormone deficiency with anatomic defect, including tumor, or with radiation.

${ }^{3} P<0.001$ for 1986 vs 2002

From 1986 to 2002, IGHD and ISS have increased whereas OGHD has decreased as a percentage of all growth hormone new starts. by year 3 and to children with IGHD by year 4 of treatment (1). Children with IGHD showed a greater response to daily dosing versus three times a week dosing (same total weekly dose). For all diagnoses with at least 7 years of data (IGHD, OGHD, ISS and Turner syndrome), height S.D. deficit continued to decrease throughout therapy (1).

\section{Outcome predictors}

Analysis of 632 naive prepubertal children (523 IGHD, 109 OGHD) found six variables which predicted $40 \%$ of the response in IGHD (age, log maximum stimulated $\mathrm{GH}$, weight adjusted for height, dosing schedule, dose and midparental height) (2). For OGHD, only three variables (pretreatment growth rate, log maximum stimulated $\mathrm{GH}$ and age) were significant, and only $20 \%$ of the response could be predicted.

\section{Demographics}

IGHD was the predominant diagnosis in 1986 and had increased as a percentage of new GH starts by 2002 (Table 2). During the same period, OGHD declined $(P<0.001)$ while ISS increased $(P<0.001)$ as a percentage of new starts (Table 2$)$ ( $P$ values calculated by paired t-test). Females remained under-represented as a percentage of new GH starts. The gap has narrowed for those with IGHD $(22 \%$ in 1986 vs $28 \%$ in 2002 , $P=0.016)$, but not for those with OGHD $(34 \%$ in 1986 vs $41 \%$ in 2002 , NS). The height deficit at start of GH therapy was significantly less by 2002 for both sexes in all major diagnostic categories except ISS for females (Table 3). Caucasian children (75\% of the US population (3)) declined as a percentage of all treated children (89\% vs $84 \%, P=0.0002)$, while Asian children $(4 \%$ of the US population (3)) increased (1\% vs $2 \%, P=0.035)$. African-American children $(12 \%$ of the US population (3)), however, remained the most under-represented group during this period, with a decline from $5 \%$ to $3 \%(P=0.0009)$.

Table 3 NCGS demographics - decline in baseline height deficit (SDS) at start of growth hormone therapy.

\begin{tabular}{lcc}
\hline Diagnosis & 1986 & 2002 \\
\hline IGHD female & $-3.3(1.5,99)$ & $-2.6^{1}(1.1,314)$ \\
OGHD female & $-2.9(1.6,101)$ & $-2.1^{1}(1.7,119)$ \\
ISS female & $-2.9(0.7,36)$ & $-2.8(0.9,122)$ \\
Turner syndrome & $-3.4(0.8,79)$ & $-2.8^{1}(0.9,188)$ \\
IGHD male & $-2.8(1.0,348)$ & $-2.2^{1}(0.9,812)$ \\
OGHD male & $-2.9(1.5,192)$ & $-2.2^{1}(1.5,173)$ \\
ISS male & $-2.9(0.8,128)$ & $-2.4^{1}(0.9,352)$ \\
\hline
\end{tabular}

${ }^{1} P<0.0011986$ vs 2002

SDS $=$ standard deviation score

S.D., $n$ for each diagnosis and year in parenthesis.

From 1986 to 2002, there has been a significant decline in baseline height deficit at the start of growth hormone therapy in children (except females with ISS). 


\section{Substudies}

Eleven substudies have been initiated under the NCGS; four remain active (substudies 9-12 in Table 4). Selected findings from two substudies are presented here.

Data from substudy 9 were collected for 955 children with Turner syndrome between 1997 and 2000 (646 retrospectively, 189 prospectively) (4). About 65\% had both an echocardiogram and renal ultrasound, while only $35 \%$ had a formal hearing examination. Published guidelines recommend that all children with Turner syndrome receive these screening tests. These findings indicate a continuing need to increase physician awareness and improve the general health care of this population.

Another study of 471 children from this substudy demonstrated the benefit of starting GH therapy at younger ages (5). With earlier GH therapy, these children showed a greater final height gain even when estrogen therapy was also begun at an earlier, more age-appropriate time.

A retrospective survey of 250 US pediatric endocrinologists described which laboratory tests they would consistently use to evaluate children referred for short stature (6). Data from substudy 8, a prospective study of short patients expected to need follow-up beyond an initial visit, were analyzed to determine which tests were actually performed (Table 5) (7). The referred children were indeed short. The mean ht SDS of the 21733 children in the study was $-2.13( \pm 0.91$ S.D.); $13 \%$ had a ht SDS of $<-3$, and $59 \%$ met the Growth Hormone Research Society criteria (8) for severe short stature (ht SDS of $<-3$ and/or mid-parental height-adjusted ht SDS of $<1.5$ ). Screening tests appear to be performed substantially less often in practice than in theory (per the survey).

Furthermore, of all patients, $38 \%$ did not return after the initial visit, and $64 \%$ of children appeared to be lost to further testing and follow-up after one or two visits regardless of the severity of short stature (7). This lack of follow-up and the discrepancies between the survey and actual practice imply either that circumstances beyond those anticipated by pediatric endocrinologists limit evaluations or that the survey data reflect ideal goals rather than true practice behavior. In either case, these data suggest that in current practice many patients with potentially treatable and significant short stature may not be getting adequate care.

Table 4 NCGS substudies.

\begin{tabular}{ll}
\hline 1. Core study & 7. Adolescent bone age \\
2. Serial GH sampling & 8. Short stature \\
3. Psychological testing & 9. Turner syndrome \\
4. Daily dosing & 10. Bone mineral density \\
5. Urinary GH testing & 11. Chronic renal insufficiency \\
6. GHBP, IGF-1, IGF BP-3 & 12. Optimal dosing in puberty \\
\hline
\end{tabular}

The core study and substudies $9,10,11$ and 12 remain active.
Although not formal substudies, analyses of subsets of patients from the NCGS can often provide new insight into the potential response of children with a variety of medical conditions. For example, an initial review of the benefit of $\mathrm{GH}$ in cystic fibrosis (9) in patients enrolled in the NCGS led to a formal randomized study $(10,11)$, which has confirmed improved growth, decreased protein catabolism and improved clinical status when GH is used in this population. Similar data reviews have suggested benefit in a variety of conditions, including Noonan syndrome (12), Aarskog syndrome (13), intrauterine growth retardation (14) and several other syndromes associated with short stature (15).

\section{Safety monitoring}

Although an observational study such as the NCGS cannot answer questions that require a randomized, controlled clinical trial design, the size of the NCGS database does permit investigation of rare adverse events that are unlikely to be detected in smaller clinical trials (16). For example, two extensive summary reviews $(16,17)$ have provided reassuring evidence that $\mathrm{GH}$ is very unlikely to be tumorigenic. Data through 1994 for 19000 GH-treated children (47000 patient years) showed no increased risk of leukemia (de novo or recurrence) or recurrent central nervous system (CNS) tumor (16). More recent data through 1998 for 33000 children (113000 patient years) have continued to show no increase in leukemia, CNS tumors or extracranial nonleukemic malignancies (17). The latest data (through January 2002) continue to show no excess of extracranial, nonleukemic malignancy (Table 6).

Concerns have been raised about the potential for $\mathrm{GH}$ to affect the rate of mole growth (18). However, a review of the NCGS safety database, including reports submitted from the US FDA MedWatch program, showed only four cases of skin cancer (19). Three cases were in children with pre-existing risk factors (irradiation in two; Gorlin syndrome in one), and one case was in an adolescent. The latter was not in excess of the expected occurrence in this age group

Table 5 Screening tests performed within 6 months on children referred to pediatric endocrinologists for short stature.

\begin{tabular}{lccc}
\hline Screening test & $\begin{array}{c}\text { All patients } \\
(n=21733)\end{array}$ & $\begin{array}{c}\text { Severe SS } \\
(n=12633)\end{array}$ & $\begin{array}{c}\text { Survey } \\
\text { physicians }\end{array}$ \\
\hline Bone age & $67 \%$ & $71 \%$ & $99 \%$ \\
Thyroid panel & $58 \%$ & $63 \%$ & $97 \%$ \\
Chemistry panel & $40 \%$ & $43 \%$ & $83 \%$ \\
IGF-1; IGFBP-3 & $41 \% ; 23 \%$ & $46 \% ; 27 \%$ & $64 \% ; 28 \%$ \\
Karyotype: female & $18 \%$ & $23 \%$ & $69 \%$ \\
\hline${ }^{1}$ Growth Hormone Research Society consensus guidelines: ht SDS $<-3$ \\
and/or mid-parental height-adjusted ht SDS < 1.5 (reference 8). \\
${ }^{2}$ Percentage of 250 pediatric endocrinologists stating in a survey that they \\
'always used' this test to screen a short child.
\end{tabular}


Table 6 Extracranial malignancies (excluding leukemia) in patients without prior risk factors who are treated with growth hormone.

\begin{tabular}{lcccc} 
Age (years) & Patient years on GH & Expected per $\mathbf{1 0 0 0 0 0}$ patient years & Expected cases & Observed cases \\
\hline $0-4$ & 8885 & 16.6 & 1.5 & 1 \\
$5-9$ & 34944 & 7.1 & 2.5 & 4 \\
$10-14$ & 63972 & 9.4 & 6.0 & 6 \\
$15-20$ & 27086 & 18.9 & 5.1 & 5 \\
$20-24$ & 527 & 32.2 & 0.2 & 0 \\
Total & 135431 & & 15.3 & 16 \\
\hline
\end{tabular}

There is no significant difference between observed and expected cases of extracranial malignancies in children without prior risk factors who were treated with growth hormone (National Cooperative Growth Study data, 1985 through January 2002).

(19). This experience, especially when combined with a cross-sectional analysis of nevi counts in GH-treated children (including those with Turner syndrome) (19), again provides practical evidence that GH therapy is unlikely to contribute to either mole count or skin cancer.

Intracranial hypertension (IHH) is known to occur in children receiving GH therapy. The NCGS database suggests this $(16,17)$, but indicates that the risks differ by diagnostic groups, with the largest risks in children with renal insufficiency $(62$ cases/1000 patient years) and Turner syndrome (3.7 cases/1000 patient years) compared with those with GHD (1.6 cases/1000 patient years) or ISS (0 cases/1000 patient years) (16). The lack of cases in children with ISS would seem to imply that a prior medical condition or hormonal deficiency may be a necessary precondition. Fortunately, IHH is almost always reversible in these cases; GH can usually be restarted at lower doses and gradually progressed back to the therapeutic dose indicated for the patient.

Slipped capital femoral epiphysis (SCFE) has also been reported in children receiving $\mathrm{GH}$ therapy. A review of 16500 children from the NCGS (through June 1994) concluded that SCFE might be more common in treated children with either organic or idiopathic GHD, renal disease or Turner syndrome (20). Interpretation of the data is limited by accurate documentation of the timing of SCFE prior to GH therapy, by the changing incidence of SCFE in the general pediatric population with age and pubertal status, and by the prevalence of other risk factors such as obesity, hypothyroidism, untreated GHD and delayed puberty. There does not seem to be any relationship between the occurrence of SCFE while on GH and sex, dose or duration of $\mathrm{GH}$, or rapid initial growth (which usually occurs in younger children who have a lower risk of SCFE). Once again, children with ISS were at lower risk than with GHD, a finding which may implicate hormonal deficiency as a precondition.

\section{Conclusions}

The NCGS has provided extensive safety and efficacy monitoring for children receiving recombinant biosynthetic GH in the United States and Canada. Userfriendly data collection software combined with a regular sharing of that data with physician providers has resulted in widespread usage, which has, in turn, increased the power and reliability of the surveillance. After almost 20 years, data from over 47000 patients representing 165000 patient years have been collected. There are over 12000 active subjects $(\sim 75 \%$ of all currently treated patients). Safety monitoring of this large population has provided reassuring evidence that leukemia (de novo or relapse), extracranial nonleukemic neoplasms and CNS tumor recurrence are not associated with GH therapy. A small risk of IHH and SCFE has been suggested, especially in children with renal disease or Turner syndrome. Both NCGS substudies and data analysis of subpopulations within the NCGS database have provided important insights into actual clinical practice and the potential therapeutic utility of GH in a variety of syndromes.

\section{References}

1 Root AW, Kemp SF, Rundle AC, Dana K \& Attie KM. Effect of longterm recombinant growth hormone therapy in children - the National Cooperative Growth Study, USA, 1985-1994. Journal of Pediatric Endocrinology and Metabolism 199811 403-412.

2 Blethen SL, Compton P, Lippe BM, Rosenfeld RG, August GP \& Johanson A. Factors predicting the response to growth hormone therapy in prepubertal children with growth hormone deficiency. Journal of Clinical Endocrinology and Metabolism $1993 \mathbf{7 6}$ 574-579.

3 United States Census 2000 Summary File 1, http://factfinder. census.gov/servlet/QTTable?_bm = y\&-geo_id = 01000US\&-qr_ name $=$ DEC_2000_SF1_U_QTP5\&-ds_name $=$ DEC_2000_SF1_ $\mathrm{U \&}$ - lang $=$ en\&- sse $=$ on

4 Parker KL, Wyatt DT, Blethen SL, Baptista J \& Price L. Screening girls with Turner syndrome: the National Cooperative Growth Study Experience. Journal of Pediatrics 2003143 133-135.

5 Reiter EO, Blethen SL, Baptista J \& Price L. Early initiation of growth hormone treatment allows age-appropriate estrogen use in Turner syndrome. Journal of Clinical Endocrinology and Metabolism 200186 1936-1941.

6 Wyatt DT, Mark D \& Slyper AH. A survey of growth hormone practices by 251 pediatric endocrinologists. Journal of Clinical Endocrinology and Metabolism $1995803292-3297$.

7 Wyatt DT, Blethen S \& Tateo J. Initial evaluation of children referred for short stature. Presented at the Genentech 14th Annual National Cooperative Growth Study Investigators Meeting, San Francisco, CA, September 2000. 
8 GH Research Society. Consensus guidelines for the diagnosis and treatment of growth hormone deficiency in childhood and adolescence: summary statement of the GH Research Society. Journal of Clinical Endocrinology and Metabolism 200085 3990-3993.

9 Hardin DS \& Sy JP. Effects of growth hormone treatment in children with cystic fibrosis: the NCGS experience. Journal of Pediatrics 1997131 S65-S69.

10 Hardin DS, Ellis KJ, Dyson M, Rice J, McConnell R \& Seilheimer DK. Growth hormone improves clinical status in prepubertal children with cystic fibrosis: Results of a randomized controlled trial. Journal of Pediatrics 2001139 636-642.

11 Hardin DS, Ellis KJ, Dyson M, Rice J, McConnell R \& Seilheimer DK. Growth hormone decreases protein catabolism in children with cystic fibrosis. Journal of Clinical Endocrinology and Metabolism $2001864424-4428$

12 Romano AA, Blethen SL, Dana K \& Noto RA. Growth hormone treatment in Noonan syndrome: the NCGS experience. Journal of Pediatrics $1996 \mathbf{1 2 8}$ S18-S21.

13 Petryk A, Richton S, Sy JP \& Blethen SL. The effect of growth hormone treatment on stature in Aarskog syndrome. Journal of Pediatric Endocrinology and Metabolism 199912 161-165.

14 Chernausek SD, Breen TJ \& Frank GR. Linear growth in response to growth hormone treatment in children with short stature associated with intrauterine growth retardation: the NCGS experience. Journal of Pediatrics $1996 \mathbf{1 2 8}$ S22-S27.
15 Rosenfeld RG \& Buckway CK. Should we treat genetic syndromes? Journal of Pediatric Endocrinology and Metabolism 200013 971-981.

16 Blethen SL, Allen DB, Graves D, August G, Moshang T \& Rosenfeld R. Safety of recombinant deoxyribonucleic acid-derived growth hormone: the National Cooperative Growth Study Experience. Journal of Clinical Endocrinology and Metabolism 199681 $1704-1710$

17 Maneatis T, Baptista J, Connelly K \& Blethen S. Growth hormone safety update from the National Cooperative Growth Study. Journal of Pediatric Endocrinology and Metabolism 200013 1035-1044

18 Bourguignon J-P, Pierard GE, Ernould C, Heinrichs C, Craen M, Rochiccioli P, Arrese JE \& Franchimont C. Effects of human growth hormone therapy on melanocytic naevi. Lancet 1993 341 1505-1506.

19 Wyatt DT. Melanocytic nevi in children treated with growth hormone. Pediatrics 1999104 1045-1049.

20 Blethen SL \& Rundle AC. Slipped capital femoral epiphysis in children treated with growth hormone. A summary of the NCGS experience. Hormone Research 199646 113-116.

Received 29 March 2004

Accepted 19 April 2004 